\title{
Defeating the Odds: Onychocryptosis, an Underestimated Cause of Morbidity
}

\section{Carlos J. Roldan ${ }^{1,2}$, María C. Correa ${ }^{3}$, Valentina Vélez ${ }^{3}$}

${ }^{1}$ Department of Pain Medicine. MD Anderson Cancer Center. The University of Texas Health Science Center, Houston, Texas.

${ }^{2}$ Department of Emergency Medicine. The University of Texas Health Science Center, Houston, Texas. ${ }^{3}$ Universidad CES, Medellin, Colombia. croldan@mdanderson.org

*Corresponding Author: Carlos J. Roldan, MD, MD Anderson Cancer Center, The University of Texas Health Science Center at Houston, 1515 Holcombe Blvd. Houston, Texas.

\begin{abstract}
Onychocryptosis or ingrown toenail is a common disorder that involves the great toe. In general OC is considered a benign pathology. The symptoms and complications originate after the nail curls in and digs into the cuticle and the soft tissues of the toe. As such, toe pain is the most common clinical manifestation resulting in missed work as well as limited activities in sports. Complications of onychocryptosis like osteomyelitis are extremely rare but has been reported in presence of risk factors such diabetes mellitus, vascular insufficiency, immunosuppression and distorted anatomy. A healthy young individual presenting to the emergency department with fever of unknown origin secondary to bilateral OC defeats all the odds and outlines the importance of a thorough history taking and physical exam. It also demonstrates that what most consider a benign pathology can also be associated to severe complications. We report a case of a healthy young male who presented to a primary care clinic with low grade fever of unknown origin. He was evaluated for third time in six weeks due to recurrent abnormal temperature, fatigue, night sweats, progressive weakness and anorexia. His HIV test was negative and his preliminary work up was non-diagnostic. He was referred to the emergency department in order to be hospitalized and undergo a cardiac ultrasound and other diagnostic tests to investigate the etiology of his fever of unknown origin. The physical exam in the emergency department revealed bilateral onychocryptosis with significant tenderness, erythema and cloudy fluid drainage. The patient claimed that his feet were never examined even though he had complained of painful ingrown toenails. Radiographs of compromised toes showed bone cortex erosion suspicious for osteomyelitis. The patient was hospitalized with intravenous antibiotics. The diagnosis was confirmed with MRI and the patient was discharged home afebrile 4 days later.
\end{abstract}

Keywords: Osteomyelitis; Onychocryptosis.

\section{INTRODUCTION}

Onychocryptosis (OC), unguis incarnates or ingrown toenail is a common acquired disorder of unclear etiology that involves the great toe. OC is considered a benign condition which most common manifestation includes localized pain, at times debilitating. Significant complications of OC including OM are very rare but have been reported before (1).

Among infectious diseases, occult OM is still considered an important cause of fever of unknown origin (FUO)
(2). Overall, OM of the lower extremities has been associated to the presence of risk factors such old age, immunosuppression, vascular insufficiency, poorly controlled diabetes mellitus and traumatic bone injury (3). In young and healthy individuals, inadequate self-care of OC might yield to chronic violation of subungual tissue and periosteum. In addition, poor hygiene facilitates bacterial colonization and if untreated, subsequent bone infection. Un-sanitized nail care tools might also play a role in the transmission of the infection between the toes. This might have 
facilitated the presence of bilateral compromise in the individual reported.

Regarding FUO, it remains a diagnostic challenge. The etiology is rarely elucidated in the emergency department (ED). With the development of more accurate diagnostic techniques and high-profile antibiotics, the causes of FUO have changed over the years. Neoplastic disorders have now displaced infectious diseases as the most common cause $(4,5)$. Thus, in search for pathology that many times requires high accuracy, precision and extensive laboratory and imaging testing is easy to undermine the need for a thorough history and physical exam by clinicians.

\section{CASE REPORT}

A 22-year-old man with no significant medical history was transferred from a satellite general practice clinic to the ED with fever of unknown origin and unspecific complaints including weakness, anorexia, night sweats and fatigue. With a concern for endocarditis he was fast-tracked to be hospitalized in order to undergo a cardiac ultrasound and other diagnostic tests. He had recently been confirmed to be HIV negative, and his PPD skin test and chest radiograph were also negative. His most recent white blood cell count was $9500 / \mu$ and his erythrocyte sedimentation rate (ESR) was 75 $\mathrm{mm} / \mathrm{h}$. His review per systems only added bilateral great toe pain from ingrown toenails. He was a poorly groomed, obese male, he was afebrile (temperature 99.2 F) with normal vital signs other than a blood pressure of $134 / 92 \mathrm{mmHg}$ and heart rate 104 beats per minute. His physical exam was grossly unremarkable except by bilateral onychocryptosis with significant tenderness, erythema and cloudy fluid drainage (figure 1). He claimed that for years he had struggled with his toes condition but pain had become constant and had noticed clear yellowish discharge staining his sacks which was not mentioned before. He also claimed that a foot examination was not done in prior medical encounters. Plain radiographs revealed bilateral great toe bone erosion suspicious for bilateral osteomyelitis (Figure 2). The new approach included blood cultures, wound swab culture and 1 gram of intravenous Vancomycin. The patient was hospitalized and an Orthopedics consult was requested. The diagnosis was later confirmed by bone biopsy and MRI imaging (figure 3). The patient was discharged home four days later with oral antibiotics and Podiatry follow up. His symptoms had improved and he was afebrile.
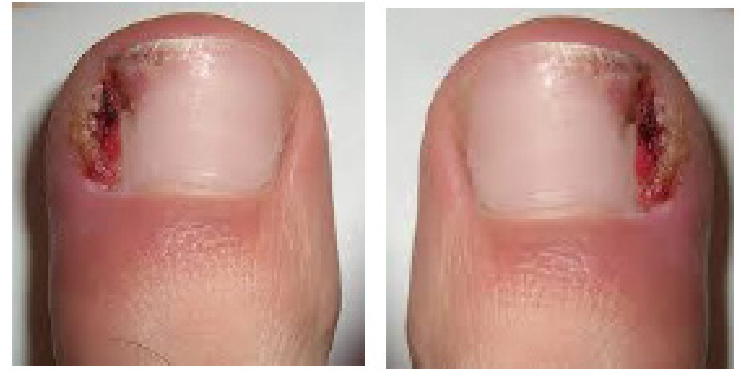

Figure 1. Onychocryptosis of bilateral great toes with no clear evidence of suppuration. There is tissue erythema, mild granulation tissue and laminar hypertrophy
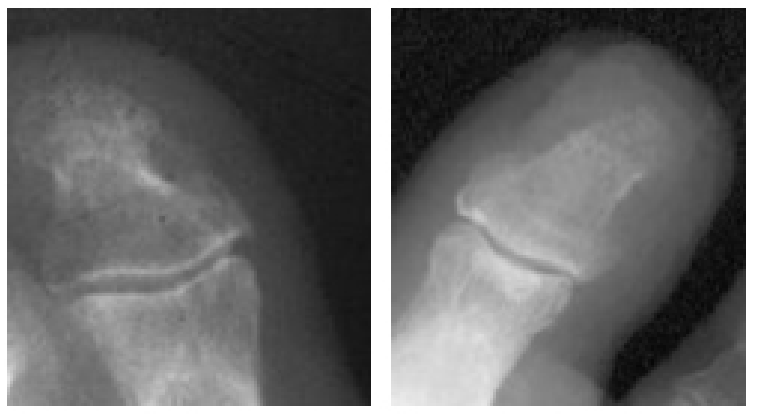

Figure 2. Localized radiograph of great toes shows cortical erosive irregularity at head of distal phalanx.
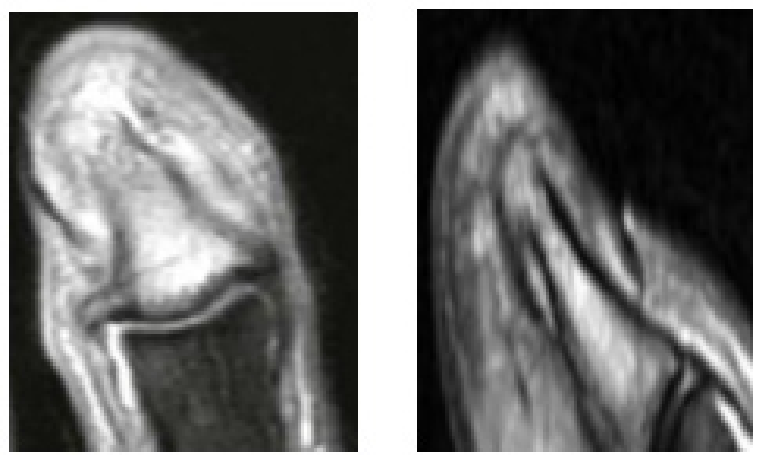

Figure 3. Axial unenhanced T1 signal within first toes with a confluent pattern localized at the head of the distal phalanx and diffuse intramedullary enhancement compatible with osteomyelitis.

\section{Discussion}

Onychocryptosis occurs when the edge of the toenail digs into, and pierces, the skin adjacent to the nail. Clinically OC manifests as an inflammatory process involving the distal portion of the toe, epithelial disruption with secondary infection and soft tissue hypertrophy (6). OC constitutes a painful disease that interferes with the quality of life by limiting sporting 
activities, school and work. Infection, if present can be originated from the normal skin flora and exacerbates the lesion as further swelling gives rise the local soft tissue pressure and the increased likelihood of penetration of the nail with eventual violation of the bony structure. The incidence of OC is higher in the pubertal age population with a male predominance of $2: 1$ to female (7). Risk factors to the development of OC include the use of some systemic chemotherapeutic drugs, subungual and periungual pyogenic granuloma caused by poorly sterilized nail trimming instruments, ill-fitting footwear, hyperhidrosis, poor foot hygiene and nail abnormalities (8). Clinical diagnosis of OC is usually evident with a careful inspection and palpation and can be classified in stages ranging from swelling to chronic deformity and destruction of the nail $(6,9)$.

$\mathrm{OM}$ as a complication of OC has rarely been described in the literature. Risk factors for this complication include localized trauma to distal phalanx of great toe, bacteremia, surgery, foreign bodies or contiguous focus infection. When OM is suspected, radiography (X rays) should be considered a screening test (1). However, bone alterations may not be detected until 5 to 7 days in children and 10 to14 days in adults (10). Majcen et al conducted a retrospective clinical study in 113 patients with symptomatic onychocryptosis and stated that almost $30 \%$ of the cases with clinical signs of infection on physical examination displayed radiologic abnormalities, predominantly periosteal reactions (11). Therefore, the value of a negative plain $\mathrm{X}$-rays is only in those cases with low probability of OM. Additionally, if OM is suspected, an advanced imaging method such as scintigraphic modalities or MRI is indicated.

In early stages, OC can be treated conservatively with thorough rising and soak of the site of the lesion and topical antiseptics, in addition to grinding/bracing the curved nail surface.Advanced stage of the pathology can be treated with non-invasive procedures such chemical ablation with phenol (12). A clinical study comparing phenolization and surgical matrix resection in 110 patients with OC showed no significant difference in terms of recurrence rate between both procedures (13). Early referral to Podiatry for management of high risk patients or those with advanced OC is always a good alternative.
It is worth noticing that clinical diagnosis, a diagnosis made on the basis of medical signs and patient-reported symptoms, rather than diagnostic tests, laboratory and diagnostic imaging seems to be underutilized. The impact of uncertainty and rising costs on the practice of medicine have implications that go beyond improvement of the quality of care. Medical societies need to expand their efforts to develop guidelines for care and to do so in an evidence-based, cost-conscious way (14).

\section{Conclusion}

Onychocryptosis is a common pathology of the great toes. Although considered benign, it can affect the quality of life. Rare complications such osteomyelitis can be overlooked. The presence of simultaneous osteomyelitis at both great toes in a healthy young individual defeats all medical odds. A medical history and physical exam seems to be an underutilized tool in decrement of accuracy of diagnosis and cost of care.

\section{REFERENCES}

[1] Akdeniz H, Ozer K, Dikmen A, Kocer U. Common surgery, uncommon complication. Dermatol Pract Concept. 2015; 5(4): 1-3.

[2] Zeller JL, Burke AE, Glass RM. JAMA patient page. Osteomyelitis. JAMA. 2008; 299: 858.

[3] Henke PK, Blackburn SA, Wainess RW, et al. Osteomyelitis of the Foot and Toe in Adults Is a Surgical Disease Conservative Management Worsens Lower Extremity Salvage. Ann Surg. 2005; 241(6): 885-894.

[4] Cunha BA, Bouyarden M, Hamid NS. Fever of unknown origin (FUO) caused by multiple myeloma: the diagnostic value of the Naprosyn test. Heart Lung. 2006; 35: 358-362.

[5] Bleeker-Rovers CP, Van der Meer JW, Oyen WJ. Fever of unknown origin. Semin Nucl Med. 2009; 39: 81-87.

[6] Maddy AJ, Tosti A. Hair and nail diseases in the mature patient. Clinics in Dermatology. 2018; 36: 159-166.

[7] Bryant A, Knox A. Ingrown toenails: the role of the GP. Aust Fam Physician. 2015; 44(3): 102-105.

[8] Jenkins DW, Cooper K, Heigh EG. Prevalence of podiatric conditions seen in Special Olympics 
Defeating the Odds: Onychocryptosis, an Underestimated Cause of Morbidity

athletes: a comparison of USA data to an international population. The Foot. 2015; 25 (1): 5-11.

[9] Tucker JR. Nail Deformities and Injuries. Prim Care. 2015; 42(4): 677-91.

[10] Pineda C,Espinosa R, Pena A. Radiographicimaging in osteomyelitis: the role of plain radiography, computed tomography, ultrasonography, magnetic resonance imaging, and scintigraphy. Semin Plast Surg. 2009; 23: 80-89.

[11] Majcen ME, Wilfinger CC, Pilhatsch A.Interpretation of radiologic abnormalities in patients with chronically infected ingrown toenails with regard to a possible exogenic osteomyelitis. J Pediatr Surg. 2009; 44: 2179-2183.
[12] Romero-Perez D, Betlloch-Mas I, Encabo-Duran B. Onychocryptosis: a long-term retrospective and comparative follow-up study of surgical and phenol chemical matricectomyin 520 procedures. International Journal of Dermatology. 2017; 56: 221-224.

[13] Herold N, Houshian S, Riegels-Nielsen P. A prospective comparison of wedge matrix resection with nail matrix phenolization for the treatment of ingrown toenail. J Foot Ankle Surg 2001; 40: 390-395.

[14] McNeil BJ. Hidden Barriers to Improvement in the Quality of Care. N Engl J Med. 2001; 345: 1612-1620.

Citation: Roldan CJ, Correa MC, Vélez V. Defeating the Odds: Onychocryptosis, an Underestimated Cause of Morbidity. Archives of Emergency Medicine and Intensive Care. 2018; 1(1): 06-09.

Copyright: (C) 2018 Roldan CJ, Correa MC, Vélez V. This is an open access article distributed under the Creative Commons Attribution License, which permits unrestricted use, distribution, and reproduction in any medium, provided the original work is properly cited. 